\title{
InGaN Microring Light-Emitting Diodes
}

\author{
H. W. Choi, C. W. Jeon, and M. D. Dawson, Senior Member, IEEE
}

\begin{abstract}
The fabrication and performance of an InGaN lightemitting diode (LED) array based on a microring device geometry is reported. This design has been adopted in order to increase the surface area for light extraction and to minimize losses due to internal reflections and reabsorption. Electrical characteristics of these devices are similar to those of a conventional large-area LED, while the directed light extraction proves to be superior. In fact, these devices are found to be more efficient when operated at higher currents. This may be attributed to improved heat sinking due to the large surface area to volume ratio. The potential applications of these devices are also discussed.
\end{abstract}

Index Terms-Extraction efficiency, gallium nitride, light-emitting diode (LED), microlight-emitting diode, microring.

$\mathbf{L}$ IGHT-EMITTING diodes (LEDs) based on InGaN semiconductor materials have become commercialized products in recent years. Nevertheless, further progress is still strongly desired in order for these devices to reach efficiency levels achievable with other III-V materials' systems. Improvements to epitaxy techniques could lead to enhanced internal quantum efficiencies, while the external quantum efficiency requires further optimization in the design of device geometry and advances in device packaging.

A major imitation to the external quantum efficiency lies in the extraction efficiency of light from the device mesa structure. As much as $90 \%$ of light emitted by the active region may be trapped as a result of the refractive index difference between $\mathrm{GaN}$ and air [1]. Various methods of addressing this shortcoming have been reported involving manipulation of the device geometry. This includes the proposal of an interdigitated mesa design [2], and the demonstration of light emission from LEDs of microdisk geometry [3], [4]. We have also reported on the enhancement of efficiency obtained from micrometer-scale emitters [5]. It is believed that the benefits derived from the structure can be attributed to the greatly enhanced surface area of the device, allowing more light to pass through the LED-air interface.

In this letter, we report on the design, fabrication, and performance of micrometer-scale GaN-based LEDs of ring geometry. The microring design is commonly used in the fabrication of optics components such as resonators, filters, and modulators [6], [7], but has not been, to the best of our knowledge, adopted for semiconductor-based emissive devices, although optical studies on InGaN microring structures have been reported [8], [9]. The benefits of the microring LEDs will be explored in this work, and their characteristics critically compared to conventional LEDs.

Manuscript received May 21, 2003; revised July 29, 2003

The authors are with the Institute of Photonics, University of Strathclyde, Glasgow G4 0NW, U.K. (e-mail: anthony.choi@strath.ac.uk).

Digital Object Identifier 10.1109/LPT.2003.818903

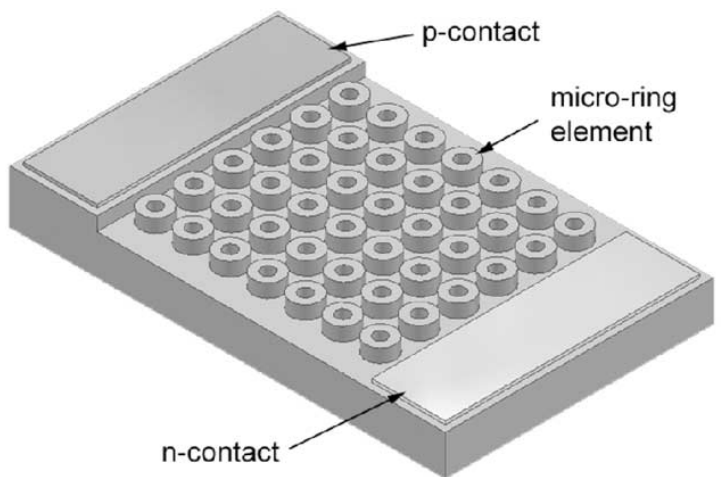

(a)

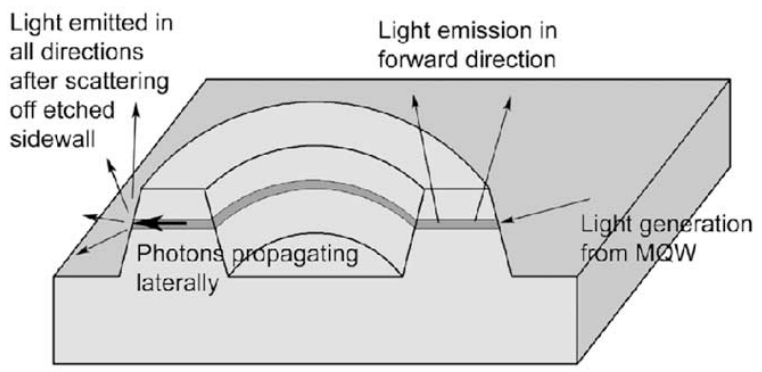

(b)

Fig. 1. (a) Schematics of the design of a microring LED and (b) diagram showing the possible light extraction pathways from a microring geometry.

The microring and conventional "broad area" geometry LED devices, each with the same total active area of $48000 \mu \mathrm{m}^{2}$, are fabricated on an LED wafer grown on the c-plane of a sapphire substrate. The LED structure consists of a $25-\mathrm{nm} \mathrm{GaN}$ buffer layer, 3.2 $\mu \mathrm{m}$ of Si-doped $\mathrm{GaN}$, a three-period $\mathrm{InGaN}-\mathrm{GaN}$ multiquantum-well targeted for emission at $\sim 470 \mathrm{~nm}$, topped with a $0.25-\mu \mathrm{m} \mathrm{Mg}$-doped $\mathrm{GaN}$ epi-layer. Activation of the $\mathrm{Mg}$ dopant was carried out by rapid thermal annealing at $950{ }^{\circ} \mathrm{C}$ for $30 \mathrm{~s}$ in a $\mathrm{N}_{2}$ ambient. Processing of the devices begins with the formation of mesa structures using inductively coupled plasma dry etching. An additional masking step was needed to pattern the microring network in the active region, whose external diameters ranged from 12 to $20 \mu \mathrm{m}$, with a typical height of $0.6 \mu \mathrm{m}$. In order to achieve conformal metal coverage across the microring structures, an isotropic etch recipe was employed, which resulted in the formation of tapered sidewalls.

Prior to metal deposition, a 40-nm-thick $\mathrm{SiO}_{2}$ layer was deposited onto the sample by electron-beam deposition, which acts as an isolation layer. The p-type contact areas are exposed by a liftoff process. Finally, the metal layers, including the spreading and pad layers, were deposited by electron-beam evaporation patterned by a liftoff procedure. The choice of metal is Ti-Al $(20 / 200 \mathrm{~nm})$ for the n-contact pad and Ni-Au (30/30 nm for spreading, 20/200 $\mathrm{nm}$ for pad) for the p-contact. A detailed schematic of the microring device is illustrated in Fig. 1(a). 


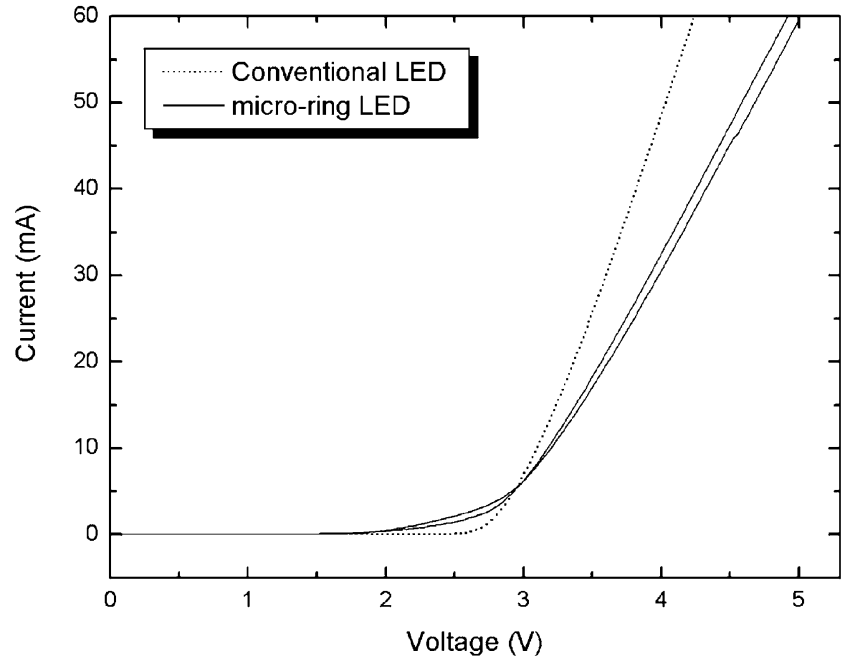

Fig. 2. $I-V$ characteristics of a microring LED compared to a conventional large-area LED.

The current-voltage $(I-V)$ characteristics of the devices were measured with an HP4155 parametric analyzer, while the light output power measurements were performed using a calibrated power meter with the Si detector (detector area $3 \times 3 \mathrm{~mm}^{2}$ ) approximately $8 \mathrm{~mm}$ above the device, collecting light emitted in the forward direction.

The turn-on and operating voltages of microring devices are marginally higher compared to the conventional LED devices, as shown from the $I-V$ characteristics in Fig. 2. This can be attributed to the effective contact area to p-type GaN [10]. The microring device is essentially an interconnected array of individual devices, each with their own ohmic contact. In each device, the effective ohmic contact is of the order of $10^{2} \mu \mathrm{m}^{2}$, about 200 times smaller than a conventional LED. Due to the granular nature of the $\mathrm{NiO}_{2}$ alloy across the metal-semiconductor interface, the effective contact area is even smaller. As a result, a larger resistive drop may be expected across the contact.

Nevertheless, a measurable increase in output power directed to the detector can be obtained from the microring LEDs. The light output-current $(L-I)$ curves, as shown in Fig. 3, reveal that the microring devices emit more light to the detector at a wide range of injection currents. While enhancement of internal quantum efficiency has been cited as the reason for the superior performance of microdisk LEDs [11], we have argued that this is not the case [5]. An increase in extraction efficiency from the mesa structure is a more plausible explanation. Since the total active area of the microring array corresponds to that of the conventional reference LED, the amount of light extracted from the top surface (through the current spreading layer) would be identical. However, the sidewalls facilitate the extraction of in-plane propagating photons, as in microdisks. The microring design, however, generates an additional (internal) sidewall for even greater light extraction efficiency. This is again evident from the $L-I$ curves of Fig. 3, where the emission capabilities of the microring are shown to be superior to the microdisk. The possible light extraction pathways are highlighted in the schematics in Fig. 1(b). Additionally, since the dimensions of the devices are

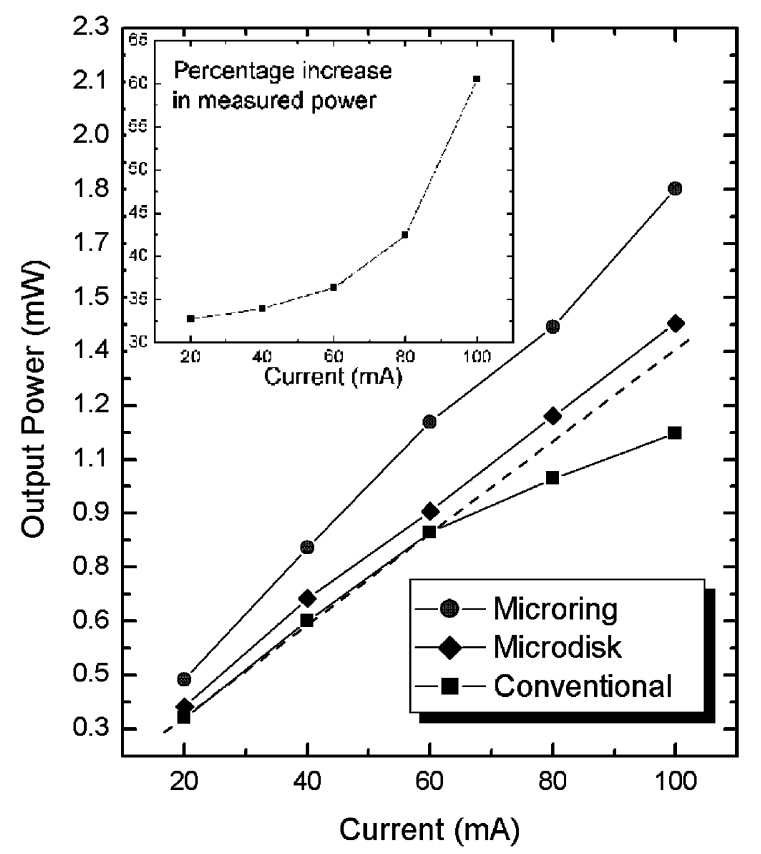

Fig. 3. $L-I$ plots for microring, microdisk and conventional LEDs. The percentage increase in measured output power of a microring LED over a conventional LED is shown in the inset.

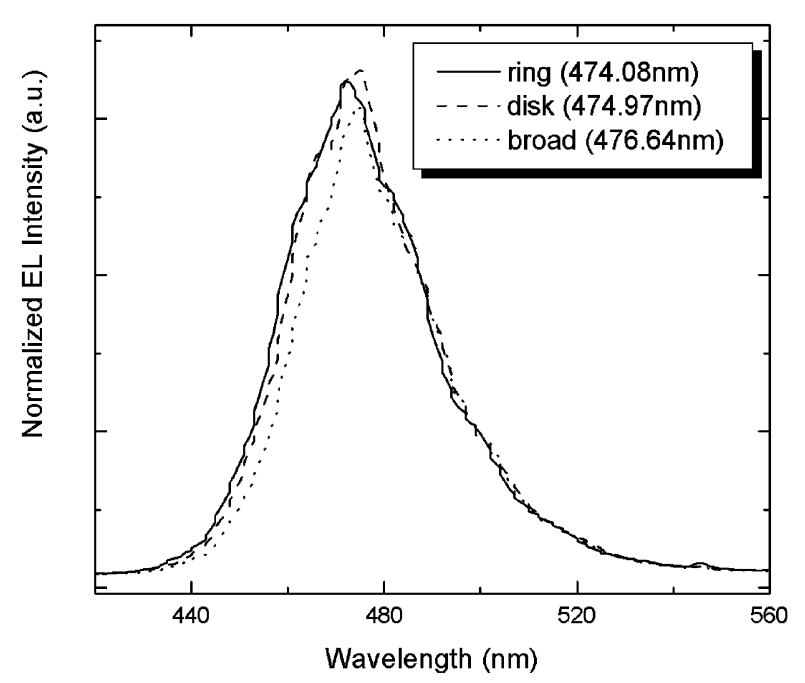

Fig. 4. Normalized EL spectra of microring, microdisk and broad-area LEDs.

on the scale of micrometers, (re)absorption can also be minimized, as the mean absorption length (at $470 \mathrm{~nm}$ ) is approximately $10 \mu \mathrm{m}$ [12].

The increase of efficiency achievable from the microring LED over the conventional LED is not constant at different injection currents. As shown in the inset of Fig. 3, this ratio is $34 \%$ at $20 \mathrm{~mA}$ and $67 \%$ at $100 \mathrm{~mA}$. This can be attributed to the resultant morphology of the devices. In a conventional LED, substantial heating of the device takes place at higher current levels ( $>50 \mathrm{~mA}$ ), where the $L-I$ curve deviates from linearity. Thus, the practical operating current is limited to typically 20 or $30 \mathrm{~mA}$. On the other hand, the large increase of surface area for light extraction also plays the important role of heat sinking. This is evident from the linearity of the $L-I$ curve for the microring LED in Fig. 3. As such, these devices 


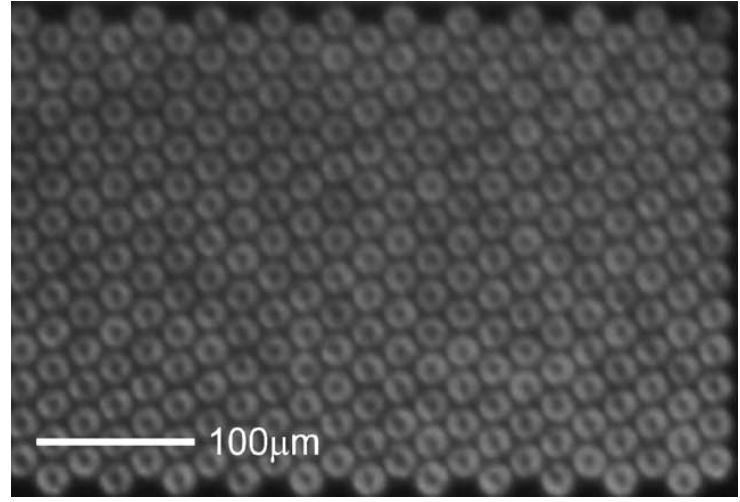

Fig. 5. A microring LED in operation, biased at $10 \mathrm{~mA}$.

can be operated in the regime of $50-80 \mathrm{~mA}$ to maximize their potential.

A 2.5-nm blueshift in the peak wavelength of the microring device in the electroluminescent (EL) spectra, shown in Fig. 4, has also been observed (compared to a broad-area LED). This can be attributed to 1) strain relaxation, 2) heating effects, 3) band-filling, or a combination of the three. However, contrary to the observations of Zeng et al. [8], resonant cavity modes, such as whispering-gallery and radial modes, are not prominent in the spectra. Consequently, spectral purity in microring LEDs is similar to the conventional LEDs. A microphotograph of a microring LED operated at $10 \mathrm{~mA}$ is illustrated in Fig. 5, which shows uniform light emission from each individual microring element across the array. Apart from the benefits of increased light extraction efficiencies, it is believed that there potentially are more implications associated with the geometry of the devices. For instance, the microrings may act as "wells" for the insertion of phosphors or polymers in the fabrication of white-light LEDs.

In conclusion, an InGaN blue LED has been fabricated with a novel microring geometry. The interconnected microring elements form a highly efficient emissive device, due to the enhancement of light extraction through the sidewalls. As much as $67 \%$ increment in light emission at the photodetector can be achieved at higher currents. The large surface area to volume ratio also helps in heat sinking, making them suitable for operation at high currents. It is proposed that the microring design may be adopted as a template for white light LEDs, where suitable phosphors or polymers may be placed into the rings for excitation.

\section{REFERENCES}

[1] I. Schnitzer, E. Yablonovitch, C. Caneau, T. J. Gmitter, and A. Scherer, " $30 \%$ external quantum efficiency from surface textured, thin-film lightemitting diodes," Appl. Phys. Lett., vol. 63, pp. 2174-2176, 1993.

[2] X. Guo, Y.-L. Li, and E. F. Schubert, "Efficiency of GaN/InGaN lightemitting diodes with interdigitated mesa geometry," Appl. Phys. Lett., vol. 79, pp. 1936-1938, 2001.

[3] H. W. Choi, C. W. Jeon, M. D. Dawson, P. R. Edwards, and R. W. Martin, "Fabrication and performance of parallel-addressed In GaN micro-LED arrays," IEEE Photon. Technol. Lett., vol. 15, pp. 510-512, Apr. 2003.

[4] S. X. Jin, J. Li, J. Y. Lin, and H. X. Jiang, "InGaN/GaN quantum well interconnected microdisk light emitting diodes," Appl. Phys. Lett., vol. 77, pp. 3236-3238, 2000.

[5] H. W. Choi, C. W. Jeon, M. D. Dawson, P. R. Edwards, R. W. Martin, and S. Tripathy, "Mechanism of enhanced light output efficiency in InGaN-based microlight emitting diodes," J. Appl. Phys., vol. 93, pp. 5978-5982, 2003.

[6] P. P. Absil, J. V. Hryniewicz, B. E. Little, P. S. Cho, R. A. Wilson, L. G. Joneckis, and P. T. Ho, "Wavelength conversion in GaAs micro-ring resonators," Opt. Lett., vol. 25, pp. 554-556, 2000.

[7] P. Rabiei, W. H. Steier, Z. Cheng, and L. R. Dalton, "Polymer micro-ring filters and modulators," J. Lightwave Technol., vol. 20, pp. 1968-1975, Nov. 2002.

[8] K. C. Zeng, L. Dai, J. Y. Lin, and H. X. Jiang, "Optical resonance modes in InGaN/GaN multiple-quantum-well microring cavities," Appl. Phys. Lett., vol. 75, pp. 2563-2565, 1999.

[9] K. S. Kim, P. R. Edwards, H. S. Kim, R. W. Martin, I. M. Watson, and M. D. Dawson, "Characterization of optical properties in micro-patterned InGaN quantum wells," Phys. Stat. Sol., vol. 228, pp. 169-172, 2001.

[10] N. Blanc, P. Gueret, P. Buchmann, K. Datwyler, and P. Vettiger, "Conductance statistics of small-area ohmic contacts on GaAs," Appl. Phys. Lett., vol. 56, pp. 2216-2218, 1990.

[11] L. Dai, B. Zhang, J. Y. Lin, and H. X. Jiang, "Comparison of optical transitions in InGaN quantum well structures and microdisks," J. Appl. Phys., vol. 89, pp. 4951-4954, 2001.

[12] O. Ambacher, W. Rieger, P. Ansmann, H. Angerer, T. D. Moustakas, and M. Stutzmann, "Sub-bandgap absorption of gallium nitride determined by photothermal deflection spectroscopy," Solid State Commun., vol. 97, pp. 365-370, 1996. 